\title{
Neurobiological Consequences of Long-Term Estrogen Therapy
}

\author{
L.K. Marriott and G.L. Wenk \\ Division of Neural Systems, Memory \& Aging, Arizona Research Laboratories, University of Arizona
}

\begin{abstract}
Postmenopausal women demonstrate an increased incidence of Alzheimer's disease (AD). Epidemiological evidence suggests that estrogen replacement therapy (ERT) may reduce the risk or delay the onset of $A D$, yet recent clinical trials found no cognitive benefits of ERT in women with mild to moderate AD. This review suggests that the timing of estrogen administration may explain these conflicting results. Chronic administration has neurobiological consequences that can affect neural and immune function, but a therapy designed to mimic the natural cycle of fluctuating hormones may more effectively slow the progression of $A D$ in postmenopausal women.
\end{abstract}

KEYWORDS-estrogen; Alzheimer's disease; luteinizing hormone (LH); immune; neuroprotection

Postmenopausal women demonstrate an increased incidence of $\mathrm{Alz}$ heimer's disease (AD), and many researchers have considered whether this heightened risk is linked to their low menopausal levels of the hormone estrogen. Indeed, epidemiological evidence suggests that postmenopausal estrogen replacement therapy (ERT) may reduce the risk and delay the onset of AD. Moreover, animal studies demonstrate that estrogen has beneficial effects on brain cell survival and cognition. Despite these positive indications, however, recent clinical trials found no benefits of ERT on cognitive function in women with mild to moderate AD (for a review, see Hogervorst, Williams, Budge, Riedel, \& Jolles, 2000). In this review, we attempt to reconcile these conflicting findings, highlighting a potential misunderstanding of estrogen's action in the brain.

\section{MENOPAUSE AND ERT}

As women enter menopause, the amount of estrogen circulating in their blood declines, eventually reaching approximately $1 \%$ of the level found in younger women. This change results in numerous physiological consequences, including cognitive dysfunction, loss of attention, mood disorders, hot flushes, and increased risk of some diseases, including AD. ERTs were developed to compensate for diminished circulating hormones, thereby alleviating the undesirable symptoms associated with menopause. When epidemiological studies

Address correspondence to Gary L. Wenk, Neural Systems, Memory \& Aging, 384 Life Sciences North, Tucson, AZ 85724-5115; e-mail: gary@nsma.arizona.edu. discovered that postmenopausal women taking estrogen had a reduced risk of developing $\mathrm{AD}$, delayed onset of $\mathrm{AD}$, and a milder progression of the disease, researchers undertook some initial studies to see whether women with AD might benefit from estrogen therapy. These early studies demonstrated improvements in attention, orientation, and mood (for a review, see Hogervorst et al., 2000), thereby instigating an extensive examination of estrogen outside its established role as a reproductive hormone. Animal studies supported the idea that short-term estrogen replacement might be beneficial by showing that cognition, neurotransmitter function, brain plasticity (the ability of brain cells to change), blood flow, and neuroprotection (the ability of nerve cells to survive a variety of toxic insults) were all enhanced by estrogen (for a review, see Norbury et al., 2003).

Three randomized, double-blind, placebo-controlled intervention studies in humans demonstrated less encouraging results regarding the effects of estrogen on the brain. In the largest of these studies, the effects of ERT were initially beneficial, much as in previous studies using smaller groups of patients and shorter treatment durations; however, over the longer term, performance on a scale measuring dementia declined significantly more among women receiving ERT than among those receiving a placebo (Mulnard et al., 2000). All three of these intervention studies concluded that ERT could not improve the cognitive abilities of women with mild to moderate AD (for a review, see Hogervorst et al., 2000). Thus, results of these clinical trials suggest that relatively short-term ERT has beneficial effects on cognitive function, but that these effects are attenuated, and possibly reversed, following much longer treatment regimens.

Investigators offered several hypotheses to reconcile the conflict in the findings of epidemiological, intervention, and animal studies. For example, intervention studies were criticized for their design and potential biases in subject selection. Researchers argued that women who typically take ERT are better educated, are healthier, and have a higher socioeconomic status than nonusers, and these factors have been associated with a reduced risk of $\mathrm{AD}$. In contrast, the women enrolled in the ERT trials already had an ongoing disease process and may have been estrogen deprived for decades prior to receiving ERT, which may have altered the effectiveness of the therapy (for reviews, see Hogervorst et al., 2000, and Toran-Allerand, 2000).

\section{CONSEQUENCES OF CHRONIC ESTROGEN}

The positive and negative effects of estrogen appear to depend on the timing of estrogen exposure. Chronic administration of estrogen 
results in neurobiological consequences that can affect both neural and immune function. Premenopausal women experience cyclic fluctuations in estrogen levels that are not mimicked in postmenopausal women by the continuous replacement of estrogen through ERT.

\section{Chronic Estrogen and Cognition}

Animal experiments have shown that deficits in working memory (the temporary retention of information needed to solve a task) are not seen immediately following surgical removal of the ovaries (ovariectomy), which removes the major source of estrogen. Instead, working memory deficits develop after longer durations of estrogen withdrawal (for a review, see Markowska \& Savonenko, 2002). Chronic administration of estrogen to ovariectomized animals does not improve their cognition if they already have detectable deficits when they begin the estrogen treatment (Markowska \& Savonenko, 2002). These results parallel the absence of cognitive benefits associated with ERT in postmenopausal women who already have cognitive deficits resulting from AD. However, estrogen levels fluctuate when constant administration is combined with injections of estrogen, and this combined treatment dramatically improved working memory in previously impaired ovariectomized animals (Markowska \& Savonenko, 2002). These findings are consistent with the hypothesis that compared with constant estrogen levels, fluctuating levels may more effectively enhance cognition in postmenopausal women (Marriott, Hauss-Wegrzyniak, Benton, Vraniak, \& Wenk, 2002).

\section{Effects on Estrogen Receptors}

Estrogen receptors are specialized proteins that respond to estrogen by initiating a variety of cellular responses. A prolonged period of menopause followed by the typical regimen of estrogen replacement can produce constant estrogen levels that may lead to a decrease in estrogen receptors' number or function (downregulation), which in turn may underlie the limited effectiveness of chronic ERT (ToranAllerand, 2000). Estrogen receptors are distributed throughout the brain and located on both brain cells (neurons and glia) and immune cells. Therefore, it is not surprising that the downregulation of these receptors can have wide-ranging and dramatic effects on many neural, endocrine, and immune processes.

\section{Chronic Estrogen Induces Reproductive Aging}

The endocrine system is tightly regulated and requires a series of precisely timed hormonal signals. These signals are typically initiated in the hypothalamus and pituitary of the brain. For example, the induction of ovulation in healthy young animals and humans begins when the hypothalamus releases bursts of luteinizing hormone-releasing hormone (LHRH). LHRH stimulates production and pulsatile release of luteinizing hormone (LH) and follicle-stimulating hormone (FSH) from the pituitary gland. In turn, LH and FSH stimulate the production of steroid hormones and ovulation from the ovaries. The steroid hormones produced by the ovaries, such as estrogen and progesterone, control the reproductive cycle by acting on the hypothalamus and pituitary (for a review, see Hung et al., 2003). Thus, hormonal signals normally function in a pulsatile and tightly regulated manner. With aging, however, there is a dysregulation in these hormonal signals. Aging rodents experience a delayed onset and attenuation of LH pulses, as well as a chronic elevation of circulating estrogen, ultimately ending hormonal cycling and signaling the reproductive aging of the animal (for a review, see Tsai \& Legan, 2001).
Continuous, long-term administration of estrogen to young ovariectomized rats suppresses the LH pulses, mimicking changes seen with normal aging (Tsai \& Legan, 2001). The suppression of the LH pulses also induces alterations in hypothalamic and pituitary function. It has been suggested that reproductive aging in the rat is influenced more by the timing and duration of estrogen exposure than by true chronological age (Hung et al., 2003). In summary, the cessation of reproductive function in animals depends on a critical pattern of chronic estrogen exposure (Desjardins, Beaudet, Meaney, \& Brawer, 1995). In turn, reproductive aging can affect neural and immune function.

\section{Irreversible Damage to the Hypothalamus}

The hypothalamus is an important region for hormonal timing and control, and chronic estrogen can irreversibly damage it. For example, studies with rodents have shown that chronic estrogen can selectively destroy certain cells in the arcuate nucleus, a subregion of the hypothalamus. Specifically, more than $60 \%$ of $\beta$-endorphin neurons (brain cells that respond to $\beta$-endorphin, a type of opiate) are destroyed (for a review, see Desjardins et al., 1995). $\beta$-endorphin and other opiates are important chemicals in the brain because they directly affect reproductive function and ovulation by strongly inhibiting the pattern of LHRH release, and subsequently LH release as well. The degeneration of $\beta$-endorphin neurons might be expected to increase LH concentrations. In fact, however, the loss of these neurons leads to compensatory changes that make the hypothalamus supersensitive to residual $\beta$-endorphin and other naturally circulating opiates, thereby resulting in persistent inhibition of LH release and inducing reproductive aging of the animal.

The aging process shares many features with this hypothalamic pathology, including deficits in $\beta$-endorphin concentrations and $\beta$-endorphin cell loss (for a review, see Desjardins et al., 1995). Persistent suppression of LHRH and LH release, therefore, may underlie aspects of reproductive aging. The consequences of reproductive aging include alterations in endocrine and immune signals.

\section{ENDOCRINE AND IMMUNE INTERACTIONS IN THE BRAIN}

There is bidirectional communication between the endocrine and immune systems within the brain. Alterations in neuroendocrine signals can modulate immune function, just as alterations in immune function can have consequences for neuroendocrine function (Reichlin, 1998). Following hypothalamic damage induced by elevated estrogen levels, microglial cells (a type of immune cell in the brain) become activated (for a review, see Hung et al., 2003), releasing chemicals that can be either beneficial or destructive, depending on their timing and termination (Akiyama et al., 2000; Reichlin, 1998). For example, activated microglial cells release inflammatory cytokines, and chronic exposure to these chemicals can contribute to pathological conditions such as AD (Akiyama et al., 2000).

\section{Interactions Affecting Neuroendocrine Function}

LHRH and LH pulses are disrupted by activation of the body's inflammatory and stress pathways. Stimulation of inflammatory and stress pathways induces elevated levels of inflammatory cytokines, thereby suppressing LHRH and LH pulses and preventing ovulation 
(Karsch, Battaglia, Breen, Debus, \& Harris, 2002). In addition, activation of the hypothalamic-pituitary-adrenal axis (the major stress pathway in the body, named for the structures it comprises) stimulates the adrenal gland to release stress hormones, including glucocorticoids, that can have consequences on other processes, such as cognition and aspects of immune function. These relationships illustrate the extensive communication between the immune and endocrine systems within the brain (for a review, see Reichlin, 1998).

\section{Interactions Affecting Neuroinflammation}

$\mathrm{AD}$ is characterized by a process of chronic neuroinflammation (for a review, see Akiyama et al., 2000), and one of the hallmarks of the neuroinflammatory response is an elevation in numbers of activated microglia. Because the brains of AD patients show evidence of inflammation and the incidence of $\mathrm{AD}$ is elevated in postmenopausal women, we recently examined how the interaction of chronic neuroinflammation and either estrogen deprivation or chronic ERT affects cognitive function and the microglial response of rodents (Marriott et al., 2002).

Our results were similar to those of Markowska and Savonenko (2002), who showed that ovariectomy did not cause behavioral impairments unless deficits were already present, in that we found no cognitive impairment after ovariectomy. However, ovariectomized animals were impaired after they were administered either chronic estrogen or a treatment that caused chronic brain inflammation. Moreover, the cognitive deficit was exacerbated when chronic estrogen and inflammatory treatments were combined, a condition analogous to a postmenopausal woman with AD receiving ERT. Although a comparison between humans and rodents must be made with caution, it is interesting that continuous, long-term estrogen therapy immediately following ovariectomy in female rats led to an impairment that paralleled the cognitive deficit recently reported in postmenopausal women with $\mathrm{AD}$ who received continuous, long-term ERT initiated decades after the onset of menopause, after cognitive deficits were already present. However, we found no impairments in naturally cycling females receiving the same chronic inflammatory treatment that produced impairments in ovariectomized animals; these results are consistent with Markowska and Savonenko's finding that fluctuating estrogen improved working memory in previously impaired ovariectomized animals.

With regard to the microglial response, ovariectomized animals with chronic neuroinflammation showed a robust increase in activated microglia that was not affected by chronic administration of estrogen. The activated microglial cells were specifically localized to brain regions that regulate the autonomic nervous system, which modulates the internal state of the animal, including stress responses (e.g., the hypothalamic-pituitary-adrenal axis) and immune function. Interestingly, intact animals with neuroinflammation had approximately half the number of activated microglia, suggesting that fluctuating gonadal hormones can provide some protection from the consequences of chronic neuroinflammation.

Taken together, these results suggest that the consequences of chronic neuroinflammation, such as cognitive impairment and microglial response, depend on the internal state (including hormone status) of the animal. First, the data support other findings showing that fluctuating levels of gonadal hormones, such as estrogen, have a neuroprotective effect. Second, these results suggest that continuous, long-term ERT given to postmenopausal women with $\mathrm{AD}$, or other diseases characterized by chronic neuroinflammation, may exacerbate existing cognitive impairments (Marriott et al., 2002).

\section{TIMING IS EVERYTHING}

Reproductive aging is a dysregulation of neuroendocrine signals that can affect neural and immune function. Continuous administration of hormones may mimic a component of this process by inducing inappropriate alterations in hypothalamic and pituitary function, such as suppression of pulsatile release of LHRH and LH. Bidirectional communication between the endocrine and immune systems may underlie aspects of the aging process, as dysfunctions in one system can have dramatic consequences in the other. These systems may work together to exacerbate dysfunctions associated with aging (for a review, see Straub, Miller, Scholmerich, \& Zietz, 2000). Therefore, continuous, long-term administration of hormones, such as estrogen alone (Mulnard et al., 2000) or in combination with progestin (Shumaker et al., 2003), is likely to have negative consequences that may impair aspects of cognition and exacerbate existing diseases characterized by neuroinflammation, such as AD.

Epidemiological and intervention studies of chronic estrogen therapy have produced conflicting results. How can estrogen have deleterious effects when administered chronically to postmenopausal women yet seem to be beneficial in epidemiological studies using the same chronic ERT regimen? The answer may lie in a close examination of the timing of the initiation of estrogen therapy. Chronic estrogen cannot improve memory if cognitive deficits already exist (Markowska \& Savonenko, 2002). It has been suggested that estrogen may decrease the risk of AD but may not alter the progression of AD if the disease process has already begun (Wise, Dubal, Wilson, Rau, \& Liu, 2001). Thus, epidemiological studies may have included women who took ERT immediately following menopause, when cognitive deficits were not yet present and processes of chronic neuroinflammation stemming from the disease had not yet begun. ERT users tend to be better educated and healthier than women who do not take ERT, so epidemiological studies may skew their results by examining women who are taking ERT for noncognitive reasons (Hogervorst et al., 2000). Still, because chronic estrogen affects neural function negatively, through its effects on estrogen receptors, $\beta$-endorphin neurons, and LH pulses, the underlying explanations for the benefits seen in epidemiological studies remain unclear. The disparate results may be due to a modulatory interaction of gonadal hormones with neuroinflammation, which can regulate the output of the autonomic nervous system and the processes it controls, such as stress regulation and immune system function.

Estrogen has complex actions on the brain that may be beneficial or detrimental, depending on the timing of exposure. Current research suggests that continuous estrogen administration for long durations may have deleterious effects on endocrine and immune function, but that such effects may be mitigated by using a therapy designed to mimic the natural cycle of fluctuating hormones. Fluctuating administration of estrogen by itself, however, may be insufficient to protect against the consequences of neuroinflammation accompanying $\mathrm{AD}$, as the physiology of young, intact animals is characterized by a more complex pattern of multiple fluctuating hormones. It may be necessary to mimic this physiology more closely in order to ameliorate the cognitive and neuroinflammatory components characteristic of AD. 


\section{Recommended Reading}

Brinton, R.D. (2001). Cellular and molecular mechanisms of estrogen regulation of memory function and neuroprotection against Alzheimer's disease: Recent insights and remaining challenges. Learning \& Memory, 8, 121133.

Cholerton, B., Gleason, C.E., Baker, L.D., \& Asthana, S. (2002). Estrogen and Alzheimer's disease: The story so far. Drugs \& Aging, 19, 405-427.

Hung, A.J., Stanbury, M.G., Shanabrough, M., Horvath, T.L., Garcia-Segura, L.M., \& Naftolin, F. (2003). (See References)

Acknowledgments - This work was supported by the U.S. Public Health Service (AG10546) and the Alzheimer's Association (IIRG-012654).

\section{REFERENCES}

Akiyama, H., Barger, S., Barnum, S., Bradt, B., Bauer, J., Cole, G.M., Cooper, N.R., Eikelenboom, P., Emmerling, M., Fiebich, B.L., Finch, C.E., Frautschy, S., Griffin, W.S., Hampel, H., Hull, M., Landreth, G., Lue, L., Mrak, R., Mackenzie, I.R., McGeer, P.L., O'Banion, M.K., Pachter, J., Pasinetti, G., Plata-Salaman, C., Rogers, J., Rydel, R., Shen, Y., Streit, W., Strohmeyer, R., Tooyoma, I., Van Muiswinkel, F.L., Veerhuis, R., Walker, D., Webster, S., Wegrzyniak, B., Wenk, G., \& Wyss-Coray, T. (2000). Inflammation and Alzheimer's disease. Neurobiology of Aging, 21, $383-421$.

Desjardins, G.C., Beaudet, A., Meaney, M.J., \& Brawer, J.R. (1995). Estrogeninduced hypothalamic beta-endorphin neuron loss: A possible model of hypothalamic aging. Experimental Gerontology, 30, 253-267.

Hogervorst, E., Williams, J., Budge, M., Riedel, W., \& Jolles, J. (2000). The nature of the effect of female gonadal hormone replacement therapy on cognitive function in post-menopausal women: A meta-analysis. Neuroscience, 101, 485-512.

Hung, A.J., Stanbury, M.G., Shanabrough, M., Horvath, T.L., Garcia-Segura, L.M., \& Naftolin, F. (2003). Estrogen, synaptic plasticity and hypothalamic reproductive aging. Experimental Gerontology, 38, 53-59.
Karsch, F.J., Battaglia, D.F., Breen, K.M., Debus, N., \& Harris, T.G. (2002). Mechanisms for ovarian cycle disruption by immune/inflammatory stress. Stress, 5, 101-112.

Markowska, A.L., \& Savonenko, A.V. (2002). Effectiveness of estrogen replacement in restoration of cognitive function after long-term estrogen withdrawal in aging rats. Journal of Neuroscience, 22, 10985-10995.

Marriott, L.K., Hauss-Wegrzyniak, B., Benton, R.S., Vraniak, P., \& Wenk, G.L. (2002). Long term estrogen therapy worsens the behavioral and neuropathological consequences of chronic brain inflammation. Behavioral Neuroscience, 116, 902-911.

Mulnard, R.A., Cotman, C.W., Kawas, C., van Dyck, C.H., Sano, M., Doody, R., Koss, E., Pfeiffer, E., Jin, S., Gamst, A., Grundman, M., Thomas, R., \& Thal, L.J. (2000). Estrogen replacement therapy for treatment of mild to moderate Alzheimer disease: A randomized controlled trial. Journal of the American Medical Association, 283, 1007-1015.

Norbury, R., Cutter, W.J., Compton, J., Robertson, D.M., Craig, M., Whitehead, M., \& Murphy, D.G. (2003). The neuroprotective effects of estrogen on the aging brain. Experimental Gerontology, 38, 109-117.

Reichlin, S. (1998). Neuroendocrinology. In J.D. Wilson, D.W. Foster, H.M. Kronenberg, \& P.R. Larsen (Eds.), Williams textbook of endocrinology (9th ed., pp. 165-248). Philadelphia: W.B. Saunders.

Shumaker, S.A., Legault, C., Rapp, S.R., Thal, L.J., Wallace, R.B., Ockene, J.K., Hendrix, S.L., Jones, B.N., Assaf, A.R., Jackson, R.D., Kotchen, J.M., Wassertheil-Smoller, S., \& Watctawski-Wende, J. (2003). Estrogen plus progestin and the incidence of dementia and mild cognitive impairment in postmenopausal women. Journal of the American Medical Association, 289, 2651-2661

Straub, R.H., Miller, L.E., Scholmerich, J., \& Zietz, B. (2000). Cytokines and hormones as possible links between endocrinosenescence and immunosenescence. Journal of Neuroimmunology, 109, 10-15.

Toran-Allerand, C.D. (2000). Estrogen as a treatment for Alzheimer disease. Journal of the American Medical Association, 284, 307-308.

Tsai, H.W., \& Legan, S.J. (2001). Chronic elevation of estradiol in young ovariectomized rats causes aging-like loss of steroid-induced luteinizing hormone surges. Biology of Reproduction, 64, 684-688.

Wise, P.M., Dubal, D.B., Wilson, M.E., Rau, S.W., \& Liu, Y. (2001). Estrogens: Trophic and protective factors in the adult brain. Frontiers in Neuroendocrinology, 22, 33-66. 